\title{
A TERNARY PULSE COMPRESSION CODE: DESIGN AND APPLICATION TO RADAR SYSTEM
}

\author{
Lei Xu and Qilian Liang \\ Department of Electrical Engineering \\ University of Texas at Arlington \\ Arlington, TX 76010
}

\begin{abstract}
In this paper, we present new developed ternary code - punctured binary sequence-pair, give its definitions and the autocorrelation properties. We also investigate Doppler shift performance of the proposed code. The significant advantages of this ternary codes over conventional pulse compression codes, such as the widely used Barker codes, are zero autocorrelation sidelobes and the longer length of the code which can be as long as 31 so far. We apply our new ternary codes to radar system for target detection and observe that our codes outperform some other conventional pulse compression codes.
\end{abstract}

Index Terms - Ternary, Phase coded waveform, Pulse compression, Radar system.

\section{INTRODUCTION}

Pulse compression, which allows a radar to simultaneously achieve the energy of a long pulse and the resolution of a short pulse without the high peak power required by a high energy short duration pulse [1], is generally used in modern radar system. The main purpose of this technique is to raise the signal to maximum sidelobe (signal-to-sidelobe) ratio to improve the target detection and range resolution abilities of the radar system. The lower the sidelobes, relative to the mainlobe peak, the better the main peak can be distinguished.

One of the basic waveform designs suitable for pulse compression is the phase-coded waveform, a long pulse of duration $T$ is divided into $N$ subpulses each of width $T_{s}$. Each subpulse has a particular phase, which is selected in accordance with a given code sequence. The pulse compression ratio equals to the number of subpulses $N=T / T_{s}$.

The criterion for selecting the subpulse phases is that all the time-sidelobe of the compressed pulse should be equal and as low as possible. One family of binary phase code widely used as a form of phase coding nowadays that can produce compressed waveforms with constant sidelobe levels equal to unity is the Barker code. It has special features with which its sidelobe structure contains the minimum energy which is theoretically possible for binary codes, and the energy is uniformly distributed among the sidelobes (the sidelobe level of the Barker codes is $1 / N^{2}$ that

This work was supported in part by U.S. Office of Naval Research (ONR) under Grant N00014-07-1-0395, N00014-07-1-1024, and National Science Foundation (NSF) under Grant CNS-0721515, CNS-0831902, and CCF-0956438.
Ting Jiang

\author{
School of Telecommunications Engineering \\ Beijing University of Posts and Telecommunication \\ Beijing 100876, China
}

of the peak signal) [2]. Unfortunately, the length $N$ of known binary and complex Barker codes is limited to 13 and 25, respectively [3], which may not be sufficient for the desired radar applications. In [4] [5], polyphase codes, with better Doppler tolerance and lower range sidelobes such as the Frank and P1 codes, the Butler-matrix derived P2 code and the linear-frequency derived $\mathrm{P} 3$ and $\mathrm{P} 4$ codes were intensively analyzed. However, the low range sidelobe of the polyphase codes can not reach the level zero either, what is more, the structure of polyphase codes is more complicated and is not easy to generate comparing with binary codes. Therefore, we propose and analyze a new ternary codepunctured binary sequence-pair, whose sidelobe level is as low as zero and the longest length of which is found 31 so far, and subsequently apply it to radar system as pulse compression waveform. The results show that the new code can be a good alternative for the current used pulse compression codes in radar system.

The rest of the paper is organized as following. Section 2 introduces the basic concept and properties of our proposed code. In Section 3, examples of punctured binary sequence-pair are given and the properties are investigated. In Section 4, the performance of our proposed code is also simulated and analyzed in radar targets detection system. In Section 5, some conclusions are drawn about our ternary code.

\section{DESIGN OF PUNCTURED BINARY SEQUENCE-PAIR}

A CW sequence is made up of $N$ bits of duration $T_{s}$. The complex envelope during one period is given by

$$
x(t)=\sum_{i=1}^{N} x_{i}\left[t-(i-1) T_{s}\right], 0 \leq t \leq N T_{s}
$$

Definition 1: A sequence-pair $(\mathbf{x}, \mathbf{y})$ is made up of two $N$-length sequences $\mathbf{x}=\left(x_{0}, x_{1}, \cdots, x_{N-1}\right)$ and $\mathbf{y}=\left(y_{0}, y_{1}, \cdots, y_{N-1}\right)$.

$$
\begin{aligned}
R_{\mathbf{x y}}(\tau) & =R_{\mathbf{x y}}\left(r T_{s}\right)=\sum_{j=0}^{N-1} x_{j} y_{(j+\tau) \bmod N}^{*} \\
0 & \leq r \leq N-1,0 \leq \tau \leq(N-1) T_{s}
\end{aligned}
$$

is called the periodic autocorrelation function of the sequence pair, while $\mathbf{x}=\mathbf{y}$, the sequence-pair $(\mathbf{x}, \mathbf{y})$ turns to be a onesequence code.

Definition 2 [6]: Sequence $\mathbf{y}=\left(y_{0}, y_{1}, \cdots, y_{N-1}\right)$ is the punc- 
tured sequence for $\mathbf{x}=\left(x_{0}, x_{1}, \cdots, x_{N-1}\right)$,

$$
y_{j}= \begin{cases}0 & \text { if } y_{j} \text { is punctured } \\ x_{j} & \text { if } y_{j} \text { is Non-punctured }\end{cases}
$$

Where $p$ is the number of punctured bits in sequence $\mathbf{x}$, suppose $x_{j} \in[-1,1], y_{j} \in[-1,0,1],(\mathbf{x}, \mathbf{y})$ is called a punctured binary sequence-pair.

Definition 3: The periodic autocorrelation of punctured sequencepair $(\mathbf{x}, \mathbf{y})$ is defined

$$
R_{\mathbf{x y}}(\tau)=R_{\mathbf{x y}}\left(r T_{s}\right)=\sum_{j=0}^{N-1} x_{j} y_{(j+r) \bmod N}^{*}, 0 \leq r \leq N-1
$$

If

$$
R_{\mathbf{x y}}(\tau)= \begin{cases}E & \tau \equiv 0 \bmod \mathrm{N} \\ 0 & \text { otherwise }\end{cases}
$$

$(\mathbf{x}, \mathbf{y})$ is called optimized punctured sequence-pair [6]. Here, $E=\sum_{j=0}^{N-1} x_{i} y_{i}=N-p$, is the energy of punctured sequencepair. Then binary sequence-pair $(\mathbf{x}, \mathbf{y})$ is called a $p$-punctured sequence-pair.

Definition 4: The balance of the sequence $\mathbf{x}$ is defined as $I=$ $\sum_{j=0}^{N-1} x_{j}=n_{p}-n_{n}$, while $n_{p}, n_{n}$ are the number of ' $+1^{\prime}$ and $'-1^{\prime}$ in $\mathbf{x}$ separately.

Assume $(\mathbf{x}, \mathbf{y})$ to be an optimized punctured binary sequencepair. Several Theorems are deduced to construct more optimized punctured sequence-pairs easily.

Theorem 1: Mapping property, if $x_{i}^{\prime}=x_{-i}, y_{i}^{\prime}=y_{-i}$, then $\left(\mathbf{x}^{\prime}, \mathbf{y}^{\prime}\right)$ is optimized punctured binary sequence-pair.

Theorem 2: Opposite to element symbol property, if $x_{i}^{\prime}=$ $-x_{i}, y_{i}^{\prime}=-y_{i}$, then $\left(\mathbf{x}^{\prime}, \mathbf{y}^{\prime}\right)$ is optimized punctured binary sequence-pair.

Theorem 3: Cyclic shift property, if $x_{i}^{\prime}=-x_{(i+u)}, y_{i}^{\prime}=$ $-y_{(i+u)}$, then $\left(\mathbf{x}^{\prime}, \mathbf{y}^{\prime}\right)$ is optimized punctured binary sequencepair.

Theorem 4: Periodically sampling property, if $x_{i}^{\prime}=-x_{k i}, y_{i}^{\prime}=$ $-y_{k i}, k$ and $N$ are relatively prime, then $\left(\mathbf{x}^{\prime}, \mathbf{y}^{\prime}\right)$ is optimized punctured binary sequence-pair.

In [6], the properties, existing necessary conditions and some constructing methods have been well studied and examples of length from 3 to 31 are presented there.

\section{PROPERTIES}

\subsection{Autocorrelation Properties}

The autocorrelation function is one of the most important properties that represent the compressed pulse in an ideal pulse compression system, since it is proportional to the matched filter response in the noise-free condition.

\section{EXAMPLE 1}

The autocorrelation property of 31-length punctured binary sequence-pair $\left(\mathbf{x}_{31}=[++++---+-+-+++----\right.$ $+--+--+++-++-]$ and $\mathbf{y}_{31}=[++++000+0+0+$ $++0000+00+00+++0++0])\left({ }^{\prime}+^{\prime}\right.$ for $^{\prime} 1^{\prime}$ and $^{\prime}-^{\prime}$ for $\left.{ }^{\prime}-1^{\prime}\right)$ shown in Fig. 1.

As it is known that a suitable criterion for evaluating code of length $N$ is the peak signal to peak signal sidelobe ratio (PSR)[7]

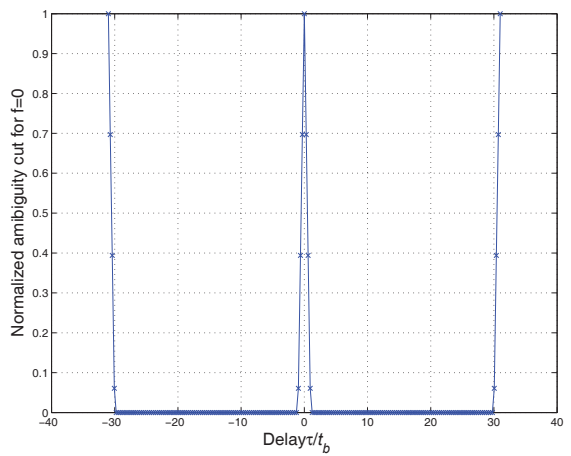

Fig. 1. Periodic autocorrelation property of 31-length punctured binary sequence-pair

of their autocorrelation function. The only aperiodic uniform phase codes that can reach the $P S R_{\max }$ are the Barker codes whose length is equal or less than 13. However, the sidelobe of the new code shown in Fig. 1 could be as low as 0 . Thus, the peak signal to peak signal sidelobe ratio can be as large as infinite. The length of the new code can expand at least to 31 that is much longer than the length of the Barker code.

\subsection{Ambiguity Function}

When the transmitted impulse is reflected by a moving target, the reflected echo signal includes a linear phase shift which corresponds to a Doppler shift $F_{D}$ [8]. As a result of the Doppler shift $F_{D}$, the main peak of the autocorrelation function is reduced as well as SNR. And the sidelobe structure is also changed thanks to the Doppler shift.

We use different codes for the transmitter and the receiver, so the single period ambiguity function of sequence-pair can be defined as:

$$
A_{T-\text { pair }}\left(\tau, F_{D}\right) \cong\left|\frac{1}{T} \int_{0}^{T} x\left(t+\frac{\tau}{2}\right) e^{j 2 \pi F_{D} t} y^{*}\left(t-\frac{\tau}{2}\right) d t\right|
$$

When the signal is of duration $M T$, the response of the correlation receiver is the PAF (periodic ambiguity function) for $M$ periods. After normalization and splitting it into $M$ sections,

$$
\begin{aligned}
A_{M T-p a i r}\left(\tau, F_{D}\right) & \cong\left|\frac{1}{M T} \int_{0}^{M T} x\left(t+\frac{\tau}{2}\right) e^{j 2 \pi F_{D} t} y^{*}\left(t-\frac{\tau}{2}\right) d t\right| \\
& =A_{T-\text { pair }}\left(\tau, F_{D}\right)\left|\frac{\sin \left(\pi F_{D} M T\right)}{M \sin \left(\pi F_{D} T\right)}\right|
\end{aligned}
$$

\section{EXAMPLE 2}

Ambiguity functions of our ternary code of length $13\left(\mathbf{x}_{13}=\right.$ $\left.[+++-++----++-], \mathbf{y}_{13}=[+0+0++0000++0]\right)$ is studied, where maximal time delay is 1 unit (normalized to length of the code, in units of $N T_{s}$ ) and maximal Doppler shift is 5 units (normalized to the inverse of the length of the code, in units of $1 / N T_{s}$ ). The ambiguity function of 13-bit long Barker code is also presented in Fig. 2 in order to compare with our ternary code of the same length. According to Fig. 2, the sidelobe of our ternary code can reach as low as zero. Nevertheless, when 


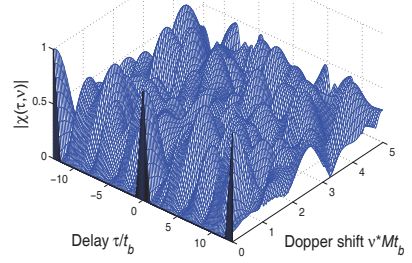

(a) Punctured binary sequence-pair

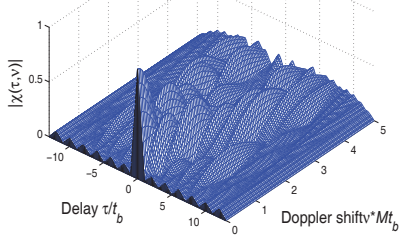

(b) Barker code
Fig. 2. Ambiguity function of 13-length codes

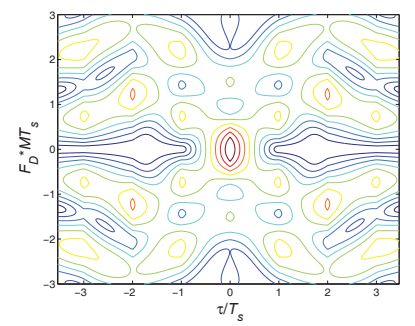

(a) $M=1$

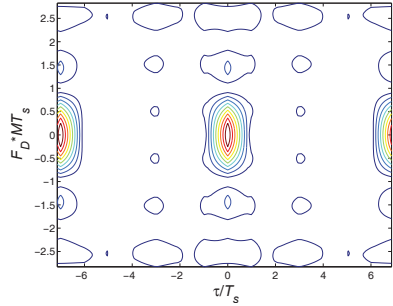

(b) $M=4$
Fig. 3. Contour plot of sequence-pair

there are Doppler shift and time delay, the ambiguity functions of our code is not as flat as those of Barker code. Our ternary code is less tolerant of Doppler shifts than Barker code. The reasons why our code is not tolerant of large Doppler shift could be that the periodic correlation property of our code is studied instead of the aperiodic correlation property.

\section{EXAMPLE 3}

In order to improve the tolerance of Doppler shift of our proposed code, we repeat the sequence-pair $M$ times to construct the signal of duration of $M T$. We study the performance of the sequencepair of $M$ periods in this section. The optimized punctured binary sequence-pair of length $7\left(x_{7}=[+++--+-], y_{7}=[++\right.$ $+00+0])$ is used here.

Figs. 3 presents contour plots of the absolute amplitudes of the ambiguity function, for the two cases $M=1,4$. The scales are normalized with respect to the bit duration of $T_{s}$. Namely, the delay axis is of $\tau / T_{s}$, and the Doppler shift axis is of $F_{D} T_{s}$. Since the single period is $T=N T_{s}$, the ambiguity function repeats itself every $N$ normalization delay units. The pronounced strips, parallel to the Doppler shift axis, appear at $N$ normalized delay units.

The corresponding 3-D plots are given in Figs. 4. The prominent feature of the ambiguity function, when $M>1$, the strips get narrower as $M$ increases. The cuts of periodic ambiguity function at $\tau / T_{s}=n N$ are independent of the number of periods $M$.

\subsection{Doppler Shift Performance Without Time Delay}

According to the previous work [8], the cut along Doppler axis is obtained as, namely, when the time delay is zero,

$$
A_{T}\left(0, F_{D}\right)=\left|\frac{\sin \left(\pi F_{D} T\right)}{\pi F_{D} T}\right|
$$

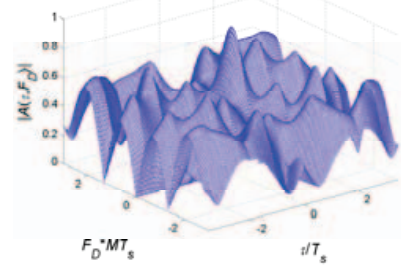

(a) $M=1$

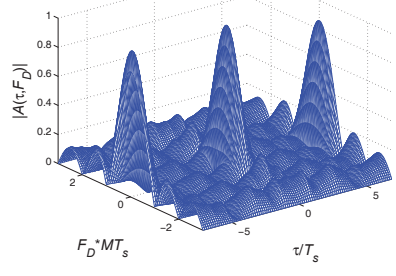

(b) $M=4$
Fig. 4. 3-D view of ambiguity function of sequence-pair $\left(x_{7}, y_{7}\right)$

It is easy to see that $F_{D}=n / T$ for all but $n=0,(n=$ $\pm 1, \pm 2, \ldots)$, the amplitudes must get a zero. It is known that Doppler frequency $F_{D}$ is given by

$$
F_{D}=2 \frac{v f_{c}}{c}
$$

where $v$ is the speed of moving target, $f_{c}$ is the carrier frequency of radar and $c$ is the speed of light. This states that Doppler shifts which equal to multiples of the PRF (pulse repetition frequency) will render the radar blind to the velocities of the targets. However, the optimized punctured sequence-pairs used here are in a different case which would be studied in this section.

The ambiguity function of single period can be simplified when there is no time delay:

$$
A_{T-\text { pair }}\left(0, F_{D}\right)=\left|\frac{1}{T} \int_{0}^{T} x(t) y^{*}(t) e^{\left(j 2 \pi F_{D} t\right)} d t\right|
$$

According to the equation (8), the ambiguity function of duration of $M$ periods could be expressed as:

$$
A_{M T-p a i r}\left(0, F_{D}\right)=A_{T-\text { pair }}\left(0, F_{D}\right)\left|\frac{\sin \left(\pi F_{D} M T\right)}{M \sin \left(\pi F_{D} T\right)}\right|
$$

Where $M$ is the number of the periods.

The Doppler shift performance without time delay is presented in Fig. 5. Without time delay, while the Doppler shift is less than 1 unit, the amplitude of our 31-length code has a sharp downward trend and decreases more quickly than $\mathrm{P} 4$ code. However, when the Doppler shift is larger than 1 unit, the performances of these codes are distinguished. On one hand, the trend presented by our 31-length code is not as regular as the other two codes. On the other hand, for P4 code, its multiples of the pulse repetition frequency will render the radar blind[1] to the velocities. Nevertheless, ambiguity values of 31-length punctured binary sequence-pair do not go to zero when Doppler frequencies are equal to multiples of the PRF. According to Fig. 5(c), $7 \times 5$-length punctured binary sequence-pair which generally resembles the 31-length P4 code is more tolerant of Doppler shift than the punctured binary sequence-pair of corresponding length 31 , but it has more ambiguity values go to zero when Doppler shift equals to some multiples of the PRF. Therefore, using the our ternary code as the compression code could, to some extent, improve the blind speed problem in moving target detection system. Using several periods of punctured binary sequence-pair could improve the tolerance of Doppler shift when Doppler shift is larger than 1 unit. 

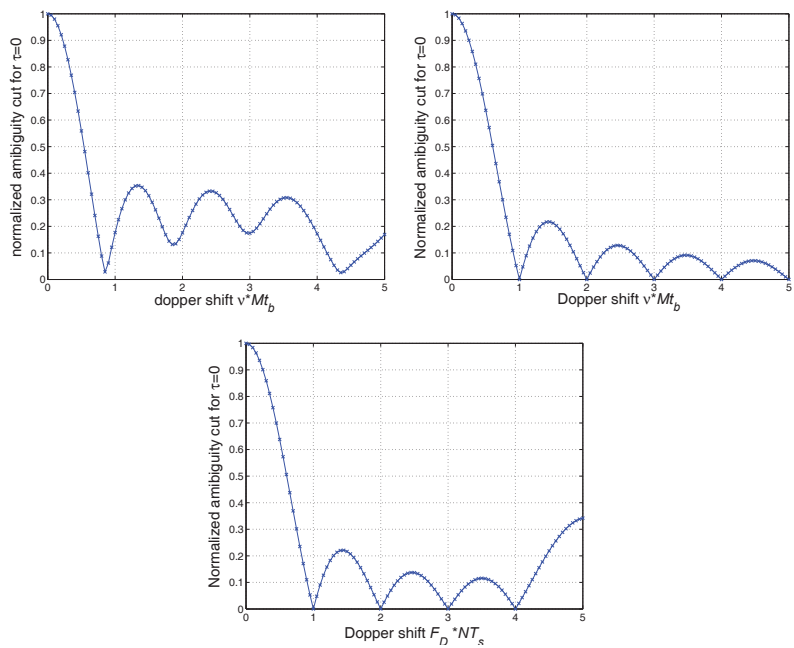

Fig. 5. Doppler shift of codes(time delay=0): (a) 31-length Punctured binary sequence-pair (b) P4 code (c) 7x5-length Punctured binary sequence-pair

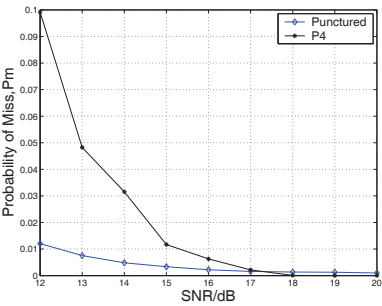

(a) $P_{m}$

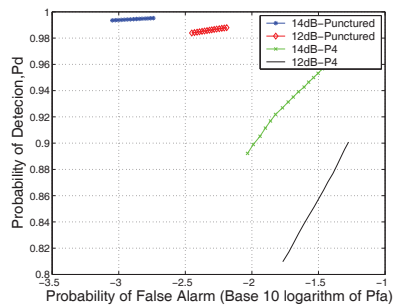

(b) $P_{d}$ versus $P_{f a}$
Fig. 6. 31-length Punctured binary sequence-pair VS. 31-length P4 code

\section{APPLICATION TO RADAR SYSTEM}

According to [8], $P_{D}$ (Probability of Detection), $P_{F A}$ (Probability of False Alarm) and $P_{M}$ (Probability of Miss) suffice to specify all of the probabilities of interest in radar system. Therefore, the above three probabilities of our newly provided ternary code in radar system are simulated, as shown in Fig. 6(a) and Fig. 6(b). The performance of 13-length Barker code and 31-length $\mathrm{P} 4$ code are also provided in order to compare with the performance of our ternary codes of corresponding lengths. In the simulation model, we ran Monte-Carlo simulation for $10^{5}$ times at each SNR value, the Doppler shift frequency which is kept less than 1 unit (normalized to the inverse of the length of the code, in units of $1 / N T_{s}$ ) is randomly given by Matlab.

In Fig. 6(a), we plotted the probabilities of miss targets detection of our 31-length ternary code and those of the same length P4 code. The probability of miss targets detection of the system using our 31-length ternary code is less than 31-length P4 code especially when the SNR is not large. When SNR is larger than $17 \mathrm{~dB}$, both probabilities of miss targets detection of the system approach zero. However, the probability of miss targets of P4 code is a little lower than our ternary code.

In addition, we also plotted the probability of detection ver- sus probability of false alarm of the coherent receiver in Fig. 6(b). Fig. 6(b) illustrates performance of our 31-length ternary codes and the same length P4 code when the SNR is $12 \mathrm{~dB}$ and $14 \mathrm{~dB}$. Having the same SNR value such as $12 \mathrm{~dB}$ or $14 \mathrm{~dB}$ in the figure, the $P_{D}$ of our 31-length ternary code is larger than $P_{D}$ of our 31-length $\mathrm{P} 4$ code while the $P_{F A}$ of the first code is also smaller than $P_{F A}$ of the latter code. In some other words, our 31-length ternary code has much higher target detection probability while keeping a lower false alarm probability. Furthermore, observe Fig. 6(b), our 31-length ternary code even has much better performance at 10dB SNR than 31-length P4 code at 14dB SNR.

\section{CONCLUSION}

A new ternary code and its properties have been investigated in this paper. The significant advantage of punctured binary sequence-pair over conventional phase compression code is the considerably reduced sidelobe as low as zero and correspondingly the significantly improved PSR. We apply the new code to the target detection in the radar system and obtain better performance. In conclusion, the punctured binary sequence-pair, which has much longer code length and better autocorrelation sidelobe property than the biphase code such as Barker code, and simpler structure than those polyphase codes such as P4 code, effectively increases the variety of candidates for pulse compression codes especially for long code.

\section{REFERENCES}

[1] S. Ariyavisitakul, N. Sollenberger, and L. Greenstein, Introduction to Radar System, Tata McGraw-Hill, 2001.

[2] J.L. Eaves and E.K. Reedy, Principles of Modern Radar, Van Nostrand Reinhold, 1987.

[3] L. Bomer and M. Antweiler, "Polyphase barker sequences," in Electronics Letters, December 1989, pp. 1577-1579.

[4] R.L. Frank, "Polyphase codes with good nonperiodic correlation properties," IEEE Transactions on Information Theory, pp. 43-45, January 1963.

[5] B.L. Lewis and F.F. Kretschuner, "Linear frequency modulation derived polyphase pulse compression codes," IEEE Transactions on Aerospace and Electronic Systems, vol. 18, pp. 637-641, Sep. 1982.

[6] T. Jiang, Research on Quasi-Perfect Binary Signal Pair and Perfect Punctured Binary Signal Pair Theory, Ph.D. thesis, Yanshan University, Qing Huangdao, 2003.

[7] M. I. Skolnik, Radar Handbook, McGraw-Hill, New York, NY, 1970.

[8] M. A. Richards, Fundamentals of Radar Signal Processing, McGraw-Hill, New York, NY, 2005. 